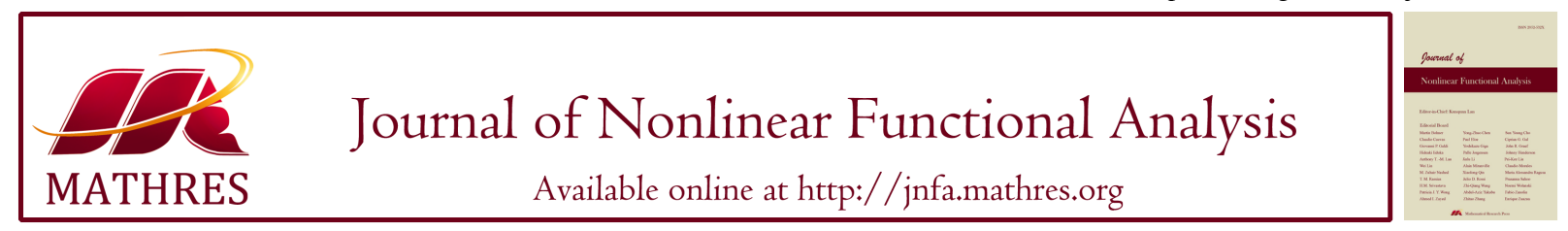

\title{
ON OPTIMALITY CONDITIONS FOR EFFICIENT SOLUTIONS IN CONSTRAINED VECTOR EQUILIBRIUM PROBLEMS IN TERMS OF STUDNIARSKI'S DERIVATIVES
}

\author{
DINH DIEU HANG ${ }^{1}$, TRAN VAN SU ${ }^{2, *}$ \\ ${ }^{1}$ Department of Basic Sciences, \\ Thai Nguyen University of Information and Communication Technology, Thai Nguyen, Vietnam \\ ${ }^{2}$ Department of Mathematics, Quang Nam University, Tam Ky, Vietnam
}

\begin{abstract}
In this paper, we study the higher-order necessary and sufficient optimality conditions for efficient solutions of vector equilibrium problems with constraints in terms of Studniarski's derivatives in real Banach spaces. We first propose the constraint qualification of the (CQm) type and then obtain the higher-order Kuhn-Tucker necessary optimality conditions for the efficient solutions, which are not required here that the ordering cone has a non-empty interior. Under suitable assumptions, the higherorder necessary optimality conditions become the higher-order sufficient optimality conditions. Second, under suitable assumptions on the nearly cone-subconvexlikeness, the higher-order Kuhn-Tucker type optimality conditions for efficient solutions in the sense of ordering cones with its interiors nonempty are derived. As applications, some the higher-order Kuhn-Tucker optimality conditions for efficient solutions of constrained vector optimization problem are also established as well. We finally provide several examples to illustrate our results.
\end{abstract}

Keywords. Vector equilibrium problem with constraints; Higher-order optimality conditions; Efficient solutions; Nearly cone-subconvexlikes; Higher-order Studniarski's derivatives.

\section{INTRODUCTION}

Fritz John and Kuhn-Tucker necessary and sufficient efficiency conditions for vector equilibrium problem with constraints and applications have been extensively investigated in the recent years by a large number of researchers; see, e.g., $[1,2,3,4,5,6,7,8,9,10,11,12]$ and the references therein. There are many tools that have been used for setting the necessary and sufficient conditions of optimality for vector equilibrium problems with set and generalized inequality constraints such as convexificators, contingent derivatives, contingent epiderivatives, directional derivatives, Radial derivatives, Radial-contingent derivatives, Studniarski's derivatives, Clarke

${ }^{*}$ Corresponding author.

E-mail addresses: dinhhangch16tn@gmail.com (D.D. Hang), suanalysis@gmail.com (T.V. Su).

Received October 10, 2019; Accepted June 25, 2020.

(C)2020 Journal of Nonlinear Functional Analysis 
and Michel-Penot subdifferentiables, subconvexlikes and nearly cone-subconvexlikes, etc, e.g. in $[13,14,15,16,17,18,19,20]$. For example, using the notations of higher-order outer and inner radial derivatives, Anh, Khanh and Tung [17] first provided several optimality conditions for particular optimization problems and then established higher-order optimality necessary conditions and sufficient ones for a general set-valued vector optimization problem with inequality constraints by making use of the notation of higher-order radial-contingent derivative. Diem, Khanh and Tung [16] first obtained optimality conditions for some particular optimization problems and then they employed this derivative along with contingent-type derivatives to analyze sensitivity for nonsmooth vector optimization by making use of the notation of nearly cone-subconvexlike. Long, Huang and Peng [9] established necessary and sufficient optimality conditions for the Henig efficient solution and the global efficient solution of vector equilibrium problem with constraints together with its application to the vector variational inequality problem and the vector optimization problem with constraints. Optimality condition for vector equilibrium problems plays an important role in nonlinear analysis and optimization theory. For the case of a constrained vector equilibrium problem with an ordering cone, which has non-empty interiors, there are a lot of results on the optimality conditions; see, e.g., [21, 22, 23, 24, 25, 26]. However, in the sense of a constrained vector equilibrium problem with an ordering cone, which has empty interiors, there are only very few results dealing with optimality conditions; see, e.g., $[4,5,6,7]$. In order to derive optimality conditions for an efficient solution of constrained vector equilibrium problem, under assumptions on the generalized convexity, Luu and Hang [10] gave Fritz John and Kuhn-Tucker necessary and sufficient optimality condition for the efficient solutions of the vector equilibrium problems with constraints in terms of Clarke subdifferentials as well as directional derivatives, which does not require that the ordering cone in the objective space has a nonempty interior. For the concept of the higher-order Studniarski derivative, we know that these derivative was established based on the different rates of change of the point under consideration in the domain space and the range space of a mapping, which are different from the higher-order derivatives based on encounter information in constraints [1,2]. It is therefore useful for establishing higher-order Kuhn-Tucker necessary and sufficient optimality conditions in terms of the higher-order Studniarski derivatives for efficient solutions in constrained vector optimization problems in Banach spaces.

Motivated by the results presented in $[1,2,4,5,6,7,9,10,11,13,14]$, we study and develop Fritz John and Kuhn-Tucker necessary and sufficient optimality conditions for efficient solutions, weak efficient solutions, Henig efficient solutions, global efficient solutions and superefficient solutions (also known as efficient solution types) of the vector equilibrium problems with set, generalized inequality and equality constraints in terms of higher-order Studniarski's derivatives in real Banach spaces. One approach in this paper is as follows. For the case of efficient solutions, we apply the separation theorem in terms of quasirelative interiors due to Cammaroto and Bella [26], which is used in sense the interiors of both sets to be empty together with the suitable constraint qualifications for receiving Kuhn-Tucker optimality conditions. For the rest efficient solutions, by making use of the separation theorem of two disjoint convex sets with nonempty interiors due to Rockafellar [27], we establish Fritz John and Kuhn-Tucker optimality conditions. Besides, we also propose the suitable constraint qualifications for obtaining Kuhn-Tucker optimality conditions. Some applications to the constrained vector optimization problem are also given. 
The remainder of the paper is organized as follows. After the introduction and some preliminaries in Section 2, we present the higher-order Kuhn-Tucker necessary and sufficient optimality conditions for the efficient solutions of constrained vector equilibrium problem with constraints in terms of Studniarski's derivatives in Banach spaces, which involve an ordering cone with an empty interior in Section 3. Section 4 deals with the higher-order necessary and sufficient optimality conditions for weak efficient solutions, Henig efficient solutions, global efficient solutions and superefficient solutions of a vector equilibrium problems with constraints. As an application, Section 5 is devoted to constructing the higher-order optimality conditions for efficient solutions of constrained vector optimization problem in terms of Studniarski's derivatives. Several examples are proposed to illustrate the results of the paper. 6, the last section, ends this paper.

\section{PRELIMINARIES}

Let $X$ be a real Banach space, $X^{*}$ be topological dual of $X, \bar{x}, v \in X$ and $m \geq 1$ be integer. Let $f$ be an extended-real-valued function defined on $X$. Taking account of Studniarski [18], the lower and upper Studniaski derivatives of order $m$ of the function $f$ at $\bar{x}$ in the direction $v$ is defined, respectively, by

$$
\begin{aligned}
& \underline{d}_{S}^{m} f(\bar{x} ; v)=\liminf _{\substack{t \rightarrow 0^{+} \\
u \rightarrow v}} \frac{f(\bar{x}+t u)-f(\bar{x})}{t^{m}}, \\
& \bar{d}_{S}^{m} f(\bar{x} ; v)=\limsup _{\substack{t \rightarrow 0^{+} \\
u \rightarrow v}} \frac{f(\bar{x}+t u)-f(\bar{x})}{t^{m}} .
\end{aligned}
$$

If $\underline{d}_{S}^{m} f(\bar{x} ; v)=\bar{d}_{S}^{m} f(\bar{x} ; v)$, one writes $d_{S}^{m} f(\bar{x} ; v)$ stands for both $\underline{d}_{S}^{m} f(\bar{x} ; v)$ and $\bar{d}_{S}^{m} f(\bar{x} ; v)$, which means that

$$
d_{S}^{m} f(\bar{x} ; v)=\lim _{\substack{t \rightarrow 0^{+} \\ u \rightarrow v}} \frac{f(\bar{x}+t u)-f(\bar{x})}{t^{m}},
$$

if this limit exists. In this case, we call $d_{S}^{m} f(\bar{x} ; v)$ the Studniaski derivative of order $m$ of the function $f$ at $\bar{x}$ in the direction $v$. For $m=1$, one writes $d_{S} f(\bar{x} ; v)$ instead of both $\underline{d}_{S}^{1} f(\bar{x} ; v)$ and $\bar{d}_{S}^{1} f(\bar{x} ; v)$. Let $\emptyset \neq C \subset X$, the interior, relative interior, closure and convex hull of $C$ be denoted by int $C, \mathrm{ri} C, \mathrm{cl} C$ and $\operatorname{co} C$, respectively. Let the cone hull of $C$ be defined by cone $C=\{t c: t \geq 0, c \in C\}$ and let the quasirelative interior of $C$ be expressed as qriC. Let the set of those $\bar{x} \in C$ such that $c l$ cone $(C-\bar{x})$ be a linear subspace of $X$. Let $Y$ and $Z$ be real Banach spaces. Let $Y^{*}$ and $Z^{*}$ be the topological dual of $Y$ and $Z$, respectively. Let and $Q$ and $S$ be two closed convex pointed cones $Y$ and $Z$, respectively. The open ball of radius $\delta>0$ around $x \in X$ in $X$ is denoted by $B(x, \delta)$. Given a vector bifunction $F: C \times C \rightarrow Y$ such that $F(c, c)=0$ for all $c \in C$, a constrainted function $g: C \rightarrow Z$, we denote by $K=\{x \in C: g(x) \in-S\}$ a feasible set.

We recall ([28]) that the contingent cone to the set $C$ at the point $\bar{x} \in \operatorname{cl} C$ is given as

$$
T(C ; \bar{x})=\left\{v \in X \mid \exists t_{n} \rightarrow 0^{+}, \exists v_{n} \rightarrow v \text { such that } \bar{x}+t_{n} v_{n} \in C, \forall n \in \mathbb{N}\right\} .
$$

In the sense that $C$ is convex (see Giorgi-Guerraggio [29] for more details),

$$
T(C ; \bar{x})=\left\{v \in X \mid \forall t_{n} \rightarrow 0^{+}, \exists v_{n} \rightarrow v \text { such that } \bar{x}+t_{n} v_{n} \in C, \forall n \in \mathbb{N}\right\} .
$$


The dual cone of the cone $Q$ is defined by

$$
Q^{+}=\left\{\xi \in Y^{*} \mid\langle\xi, q\rangle \geq 0 \quad \forall q \in Q\right\},
$$

where $\langle\cdot, \cdot\rangle$ is the coupling between $Y^{*}$ and $Y$. The quasi-interior of $Q^{+}$is denoted by $Q^{\sharp}$, i.e.,

$$
Q^{\sharp}=\left\{\xi \in Y^{*} \mid\langle\xi, q\rangle>0 \forall q \in Q \backslash\{0\}\right\} .
$$

Considering that the convex set $B$ is a base of $Q$ (i.e., $0 \notin c l B$ and $Q=\operatorname{cone} B$ ), we set

$$
Q^{\Delta}(B):=\left\{\xi \in Q^{\sharp} \mid \exists t>0 \text { such that }\langle\xi, b\rangle \geq t \forall b \in B\right\} .
$$

As we know, if $Q$ has a base $B$, then $Q$ is pointed. So, $Q^{\sharp} \neq \emptyset$. Let $B$ be a base of $Q$. It means that $0 \notin \mathrm{clB}$. Using the separation theorem in Rockafellar [27] for the two disjoint nonempty convex sets $\{0\}$ and $\operatorname{cl} B$, we have that there exists $\xi \in Y^{*} \backslash\{0\}$ such that

$$
r=\inf \{\langle\xi, b\rangle: b \in B\}>\langle\xi, 0\rangle=0 .
$$

We define an open absolutely convex neighborhood of the origin in $Y$ by

$$
V_{B}=\left\{y \in Y||\langle\xi, y\rangle \mid<\frac{r}{2}\right\} .
$$

The notation $V_{B}$ will be used throughout this paper. In this sense, for every convex neighborhood $U$ of the origin in $Y$ such that $U \subset V_{B}$, it is known in Gong' result [4] that cone $(U+B)$ is pointed and convex with $Q \backslash\{0\} \subset$ int cone $(U+B)$. In what follows, for the sake of convenience, for every $\bar{x} \in K$, one denotes as $F_{\bar{x}}()=.F(\bar{x},):. C \rightarrow Y$ and $F_{\bar{x}}(K)=\bigcup_{x \in K} F_{\bar{x}}(x)$.

We consider the following vector equilibrium problem with set and cone constraints (VEPC): Finding a vector $\bar{x} \in K$ such that

$$
F_{\bar{x}}(K) \cap(-\operatorname{int} Q)=\emptyset .
$$

The vector, which $\bar{x}$ solves (2.1) is called a weak efficient solution to problem (VEPC).

Definition 2.1. (Gong $[4,5]$ ) The vector $\bar{x}$ is called an efficient solution to problem (VEPC) iff $\bar{x}$ satisfies

$$
F_{\bar{x}}(K) \cap(-Q \backslash\{0\})=\emptyset .
$$

Definition 2.2. (Gong $[4,5]$ ) The vector $\bar{x} \in K$ is called a global efficient solution to problem (VEPC) iff there exists a pointed convex cone $H \subset Y$ with $Q \backslash\{0\} \subset \operatorname{int} H$ satisfying

$$
F_{\bar{x}}(K) \cap(-H \backslash\{0\})=\emptyset .
$$

Definition 2.3. (Gong [4, 5]) The vector $\bar{x} \in K$ will be called a Henig efficient solution to problem (VEPC), iff there exists some absolutely convex neighborhood $U$ of the origin with $U \subset V_{B}$ satisfying

$$
\text { cone }\left(F_{\bar{x}}(K)\right) \cap(- \text { int cone }(U+B))=\emptyset .
$$

Definition 2.4. (Gong $[4,5]$ ) The vector $\bar{x} \in K$ is called a superefficient solution to problem (VEPC) iff, for each neighborhood $V$ of the origin, there exists some neighborhood $U$ of the origin satisfying

$$
\text { cone }\left(F_{\bar{x}}(K)\right) \cap(U-Q) \subset V \text {. }
$$


In (2.1)-(2.5), replacing $K$ by $K \cap B(\bar{x}, \delta)$ for some $\delta>0$, we obtain the notations of local efficient, local weak efficient, local Henig efficient, local global efficient and local superefficient solutions to problem (VEPC), respectively.

We here mention that the following implications hold:

If $\bar{x}$ is a (local), either efficient solution or global efficient solution to problem (VEPC),

then $\bar{x}$ is a (local) weak efficient solution in the sense $\operatorname{int} Q \neq \emptyset$.

If $\bar{x}$ is a (local) superefficient solution to problem (VEPC),

then $\bar{x}$ is a (local) Henig efficient solution in the sense the cone Q has a base B.

If $\bar{x}$ is a (local) Henig efficient solution to problem (VEPC),

then $\bar{x}$ is a (local) superefficient solution in the sense the cone $\mathrm{Q}$ has a bounded closed base B.

According to Gong $[4,5])$, if the cone $Q$ has a bounded closed base $B$, then $\operatorname{int}\left(Q^{+}\right)=Q^{\Delta}(B)$, where one writes $\operatorname{int}\left(Q^{+}\right)$instead of the interior of $Q^{+}$with respect to the strong topology on $Y^{*}$, which is denoted by $\beta\left(Y^{*}, Y\right)$. The sets

$$
\omega=\left\{\bigcap_{i=1}^{n}\left\{y^{*} \in Y^{*}: \sup _{a \in A_{i}}\left|\left\langle y^{*}, a_{i}\right\rangle\right|<\varepsilon\right\}: A_{i}(i=1,2, \ldots, n)\right.
$$

are bounded subsets of $Y, \varepsilon>0, n \geq 1, n$ is integer $\}$

form a base of neighborhoods of the origin of the dual space $Y^{*}$ of $Y$ with respect to $\beta\left(Y^{*}, Y\right)$.

We mention that all the preceding efficient solutions are coincide in the sense $Y=\mathbb{R}$ and $Q=\mathbb{R}_{+}:=[0,+\infty)$. In order to derive optimality conditions for the efficient solution of problem (VEPC) in the condition which does not require a cone to have nonempty interiors, researchers used the following separation theorem of Cammaroto and Bella [26] in terms of quasirelative interiors (see [10] for more details).

Proposition 2.5. [26] Let $A$ and $B$ be nonempty convex subsets of $X$. Assume, in addition, that qri $A \neq \emptyset$, qriB $\neq \emptyset$ and cl cone $(q r i A-q r i B)$ is not a linear subspace of $X$. Then there exists $\xi \in X^{*}$ with $\xi \neq 0$ such that

$$
\langle\xi, x\rangle \leq\langle\xi, y\rangle, \forall x \in A, y \in B .
$$

The notation of the quasirelative interior was introduced by Borwein and Lewis [25], which is an extension of the relative interior in the sense of finite-dimensional spaces. Moreover, qriC= riC holds for all convex set $C \subset \mathbb{R}^{n}$. We next recall the concept of the nearly $Q$-subconvexlike, which will be needed in the next sections.

Definition 2.6. [9] A $Y$-valued function $f$ defined on $C$ is said to be nearly $Q$ - subconvexlike on $C$ if $\operatorname{cl}($ cone $(f(C)+Q))$ is convex.

Remark 2.7. If $f$ is $Q$-convex on a convex set $C$, that is, for any $x_{1}, x_{2} \in C$ and $t \in[0,1]$,

$$
t f\left(x_{1}\right)+(1-t) f\left(x_{2}\right) \in f\left(t x_{1}+(1-t) x_{2}\right)+Q,
$$

then $f(C)+Q$ is a convex set. So, it is nearly $Q-$ subconvexlike on $C$. The converse case is not true, in general, and the convexity of $C$ is not required in Definition 2.6. 
Finally, to obtain the Kuhn-Tucker necessary optimality conditions for problem (VEPC), we introduce the following constraint qualification of the (CQm) type:

$$
\forall \eta \in N(-S, g(\bar{x})) \backslash\{0\} \exists v_{0} \in T(C ; \bar{x}) \text { such that } d_{S}^{m}\left(\eta_{0} g\right)\left(\bar{x} ; v_{0}\right)<0 .
$$

\section{HIGHER-ORDER OPTIMALITY CONDITIONS FOR AN EFFICIENT SOLUTION}

In this section, making use of the separation theorem of Cammaroto and Bella [26] and the notion of quasirelative interiors, we can derive a Higher-order necessary and sufficient condition for an efficient solution of the vector equilibrium problem with constraints in terms of Studniarski's derivatives. Hereafter, a Higher-order necessary optimality condition will be derived.

Theorem 3.1. (Higher-order necessary optimality condition) Let $\bar{x}$ be an efficient solution to problem (VEPC). Assume that there exists the Studniarski derivative of order $m$ of $\lambda_{0} F_{\bar{x}}(\forall \lambda \in$ $\left.Q^{+}\right)$and $\eta_{0} g(\forall \eta \in N(-S, g(\bar{x})))$ at $\bar{x}$ in all the directions $v \in T(C ; \bar{x})$, qri $\left(F_{\bar{x}}, g\right)(C) \neq \emptyset$, $\operatorname{qri}(Q \times S) \neq \emptyset$ and cl cone $\left(\operatorname{qri}\left(\operatorname{co}\left(F_{\bar{x}}, g\right)(C)\right)+\operatorname{qri}(Q \times S)\right)$ is not a linear subspace of $Y \times Z$. Then there exist $(\lambda, \eta) \in Y^{*} \times Z^{*} \backslash\{(0,0)\}$ satisfying

$$
\begin{gathered}
\lambda \in Q^{+}, \eta \in N(-S, g(\bar{x})) ; \\
\min _{v \in T(C ; \bar{x})}\left\{d_{S}^{m}\left(\lambda_{0} F_{\bar{x}}+\eta_{0} g\right)(\bar{x} ; v)\right\}=0 .
\end{gathered}
$$

Moreover, $\lambda \neq 0$ under the constraint qualification of the (CQm) type.

Proof. It follows from the assumptions that qri $(-(Q \times S)) \neq \emptyset$ and $\operatorname{qri}\left(\operatorname{co}\left(F_{\bar{x}}, g\right)(C)\right) \neq \emptyset$ because $\operatorname{qri}\left(F_{\bar{x}}, g\right)(C) \neq \emptyset$, $\operatorname{qri}(Q \times S) \neq \emptyset$ and $\operatorname{qri}(Q \times S)=-\operatorname{qri}(-(Q \times S))$. By virtue of the separation theorem of Cammaroto and Bella [26], we have that there exist $(\lambda, \eta) \in Y^{*} \times Z^{*}$, not all zero, such that

$$
\langle\lambda, y\rangle+\langle\eta, z\rangle \leq\langle\lambda, a\rangle+\langle\eta, b\rangle \forall y \in-Q, z \in-S,(a, b) \in \operatorname{co}\left(F_{\bar{x}}, g\right)(C) .
$$

Combined (3.3) with $\left(F_{\bar{x}}, g\right)(C) \subset \operatorname{co}\left(F_{\bar{x}}, g\right)(C)$ yields that

$$
\langle\lambda, y\rangle+\langle\eta, z\rangle \leq\left\langle\lambda, F_{\bar{x}}(x)\right\rangle+\langle\eta, g(x)\rangle \forall y \in-Q, z \in-S, x \in C .
$$

In (3.4), we first pick $y=0, z=0$, and one second takes $y=0, x=\bar{x}$ and $z=2 g(\bar{x})$. It results in

$$
\begin{gathered}
0 \leq\left\langle\lambda, F_{\bar{x}}(x)\right\rangle+\langle\eta, g(x)\rangle \forall x \in C, \\
\langle\eta, g(\bar{x})\rangle \leq 0 .
\end{gathered}
$$

For the case $x=\bar{x}$ in (3.5), it ensures that

$$
\langle\eta, g(\bar{x})\rangle \geq 0 \text {. }
$$

Combining (3.6)-(3.7), we have the following equality holds true

$$
\langle\eta, g(\bar{x})\rangle=0 \text {. }
$$

In addition, using inequality (3.4), we obtain the following result

$$
\langle\lambda, y\rangle+\langle\eta, z\rangle \geq 0 \forall y \in Q, z \in S,
$$


which ensures that $\lambda \in Q^{+}$and $\eta \in S^{+}$with $\langle\eta, g(\bar{x})\rangle=0$. One one hand, according to Jiménez and Novo's result in [28], we obtain as follows

$$
N(-S, g(\bar{x}))=\left\{\eta \in S^{+}:\langle\eta, g(\bar{x})\rangle=0\right\},
$$

which ensures that $\eta \in N(-S, g(\bar{x}))$. On the other hand, from the inequalities in (3.5), (3.8), $F_{\bar{x}}(\bar{x})=0$ and the fact that $\bar{x}$ is an efficient solution of problem (VEPC), it follows that $\bar{x}$ is a solution of the following scalar optimization problem

$$
\text { (P) } \min \left(\lambda_{0} F_{\bar{x}}+\eta_{0} g\right)(x) \text { subject to } x \in C,
$$

where

$$
\left(\lambda_{0} F_{\bar{x}}+\eta_{0} g\right)(x)=\left\langle\lambda, F_{\bar{x}}(x)\right\rangle+\langle\eta, g(x)\rangle
$$

and

$$
\left(\lambda_{0} F_{\bar{x}}+\eta_{0} g\right)(\bar{x})=0 .
$$

We now denote by $t_{n} \rightarrow 0^{+}$the sequence of positive real numbers $\left(t_{n}\right)_{n \geq 1}$ with limit 0 and by $\left(t_{n}, v_{n}\right) \rightarrow\left(0^{+}, v\right)$ for $t_{n} \rightarrow 0^{+}$and $v_{n} \rightarrow v$ as $n \rightarrow+\infty$. For every $v \in T(C ; \bar{x})$, there exist $\left(t_{n}, v_{n}\right) \rightarrow\left(0^{+}, v\right)$ such that $\bar{x}+t_{n} v_{n} \in C \forall n \geq 1$. This together with the fact that $\bar{x}$ is a solution of problem $(\mathrm{P})$ holds that

$$
\lambda_{0} F_{\bar{x}}\left(\bar{x}+t_{n} v_{n}\right)+\eta_{0} g\left(\bar{x}+t_{n} v_{n}\right) \geq 0 \forall n \geq 1 .
$$

Combined this with the fact that $\left(\lambda_{0} F_{\bar{x}}+\eta_{0} g\right)(\bar{x})=0$, it leads to the following result

$$
d_{S}^{m}\left(\lambda_{0} F_{\bar{x}}+\eta_{0} g\right)(\bar{x} ; v)=\lim _{n \rightarrow+\infty} \frac{\left(\lambda_{0} F_{\bar{x}}+\eta_{0} g\right)\left(\bar{x}+t_{n} v_{n}\right)-\left(\lambda_{0} F_{\bar{x}}+\eta_{0} g\right)(\bar{x})}{t_{n}^{m}} \geq 0,
$$

and these yields that (3.2) is satisfied. To finish the proof, it can be seen that $\lambda \neq 0$ under the constraint qualification of the (CQm) type. Indeed, if not, then $\lambda=0$. So, $\eta \in N(-S, g(\bar{x})) \backslash$ $\{0\}$. Under the constraint qualification of the (CQm) type, there exists at least a direction $v_{0} \in$ $T(C ; \bar{x})$ such that $d_{S}^{m}\left(\eta_{0} g\right)\left(\bar{x} ; v_{0}\right)<0$. Consequently,

$$
\min _{v \in T(C ; \bar{x})}\left\{d_{S}^{m}\left(\lambda_{0} F_{\bar{x}}+\eta_{0} g\right)(\bar{x} ; v)\right\} \leq d_{S}^{m}\left(\eta_{0} g\right)\left(\bar{x} ; v_{0}\right)<0
$$

which is a contradiction. This completes the proof.

Remark 3.2. Similarly, we assert that the result obtained in Theorem 3.1 is still true for the case that $\bar{x}$ is a local efficient solution to problem (VEPC).

The next example is provided to illustrate the result obtained in Theorem 3.1.

Example 3.3. Let $X=Y=\mathbb{R}^{3}, Z=\mathbb{R}^{2}, C=[0,1] \times[0,1] \times[0,1], Q=\mathbb{R}_{+}^{2} \times\{0\}, S=\mathbb{R}_{+}^{2}$ and $\bar{x}=(0,0,0)$. Let $F_{\bar{x}}: \mathbb{R}^{3} \rightarrow \mathbb{R}^{3}$ be defined as $F_{\bar{x}}=\left(f_{1}, f_{2}, f_{3}\right)$, where

$$
f_{1}(x)=x_{1}^{m}-x_{2}^{m}+x_{3}^{m}, f_{2}(x)=x_{1}^{m} x_{2}+x_{2}^{m-2} x_{3}^{2}, f_{3}(x)=-x_{2}^{m}+x_{3}^{m}
$$

for all $x=\left(x_{1}, x_{2}, x_{3}\right) \in \mathbb{R}^{3}$. Let $g: \mathbb{R}^{3} \rightarrow \mathbb{R}^{2}$ be defined as $g=\left(g_{1}, g_{2}\right)$, where

$$
g_{1}(x)=x_{1}^{m}\left(x_{2}-1\right), g_{2}(x)=x_{2}^{m}\left(x_{3}-1\right)
$$

for all $x=\left(x_{1}, x_{2}, x_{3}\right) \in \mathbb{R}^{3}$. Then $Q^{+}=\mathbb{R}_{+}^{2} \times \mathbb{R}, N(-S, g(\bar{x}))=\mathbb{R}_{+}^{2}, T(C, \bar{x})=\mathbb{R}_{+}^{3}$. It can be easily seen that for all $v=\left(v_{1}, v_{2}, v_{3}\right) \in T(C ; \bar{x}), \lambda=\left(\lambda_{1}, \lambda_{2}, \lambda_{3}\right) \in Q^{+}$and $\eta=\left(\eta_{1}, \eta_{2}\right) \in$ 
$N(-S, g(\bar{x}))$, the higher-order Studniarski derivatives $D_{S}^{m}\left(\lambda_{0} F_{\bar{x}}\right)(\bar{x} ; v)$ and $D_{S}^{m}\left(\eta_{0} g\right)(\bar{x} ; v)$ exist and these are respectively given as

$$
\begin{aligned}
& D_{S}^{m}\left(\lambda_{0} F_{\bar{x}}\right)(\bar{x} ; v)=\lambda_{1}\left(v_{1}^{m}-v_{2}^{m}+v_{3}^{m}\right)+\lambda_{2} v_{2}^{m-2} v_{3}^{2}+\lambda_{3}\left(-v_{2}^{m}+v_{3}^{m}\right) ; \\
& D_{S}^{m}\left(\eta_{0} g\right)(\bar{x} ; v)=-\eta_{1} v_{1}^{m}-\eta_{2} v_{2}^{m} .
\end{aligned}
$$

It is easy to see that $\operatorname{qri}\left(F_{\bar{x}}, g\right)(C)=(-1,2) \times(0,2) \times(-1,1) \times(-1,0) \times(-1,0) \neq \emptyset$ and $\operatorname{qri}(Q \times S)=\mathbb{R}_{++}^{2} \times\{0\} \times \mathbb{R}_{++}^{2} \neq \emptyset$, where $\mathbb{R}_{++}^{2}=\operatorname{int}\left(\mathbb{R}_{+}^{2}\right)=\left\{\left(x_{1}, x_{2}\right) \in \mathbb{R}^{2}: x_{1}>0, x_{2}>0\right\}$. It is also evident that $\operatorname{clcone}\left(\operatorname{qri}\left(\operatorname{co}\left(F_{\bar{x}}, g\right)(C)\right)+\operatorname{qri}(Q \times S)\right)=\mathbb{R} \times \mathbb{R}_{+} \times \mathbb{R}^{3}$ is not a linear subspace of $\mathbb{R}^{5}$ and the constraint qualification of the (CQm) type is fulfilled. We mention that $\bar{x}=(0,0,0)$ is an efficient solution to the following vector equilibrium problem: Finding a vector $\bar{x} \in K$ such that

$$
F_{\bar{x}}(K) \cap(-Q \backslash\{0\})=\emptyset,
$$

in which $K=C$. This allows us to conclude that all the assumptions of Theorem 3.1 are valid. In fact, it is evident that for $\lambda=(2,1,-2)$ and $\eta=(1,0)$ :

$$
d_{S}^{m}\left(\lambda_{0} F_{\bar{x}}+\eta_{0} g\right)(\bar{x} ; v)=v_{1}^{m}+v_{2}^{m-2} v_{3}^{2} \geq 0 .
$$

In what follows, a sufficient optimality condition for the local efficient solution of problem (VEPC) can be expressed as follows.

Theorem 3.4. (Higher-order sufficient optimality condition) Let $\bar{x} \in K$ and assume that there exists the Studniarski derivative of order $m$ of $\lambda_{0} F_{\bar{x}}\left(\forall \lambda \in Q^{+}\right)$and $\eta_{0} g(\forall \eta \in N(-S, g(\bar{x})))$ at $\bar{x}$ in all the directions $v \in T(C ; \bar{x})$ and $\operatorname{dim} X<+\infty$. Suppose also that for all $v \in T(C ; \bar{x}) \backslash\{0\}$ there exist $\lambda \in Q^{+}$, a $\rceil N(-S, g(\bar{x}))$ satisfying

$$
d_{S}^{m}\left(\lambda_{0} F_{\bar{x}}+\eta_{0} g\right)(\bar{x} ; v)>0 .
$$

Then $\bar{x}$ is a local efficient solution of problem (VEPC).

Proof. Suppose that there exist $\lambda \in Q^{+}$and $\eta \in N(-S, g(\bar{x}))$ such that (3.11) is fulfilled. Then we need to show that the vector $\bar{x} \in K$ is a local efficient solution for problem (VEPC). In fact, if not, then, for each natural number $n \geq 1$, there exists a $x_{n} \in K \cap B\left(\bar{x}, \frac{1}{n}\right) \backslash\{\bar{x}\} \subset C \cap B\left(\bar{x}, \frac{1}{n}\right)$ such that

$$
F_{\bar{x}}\left(x_{n}\right) \in-Q \backslash\{0\} .
$$

Using this result, we can find a sequence $\left(x_{n}\right)_{n \geq 1} \subset K \backslash\{\bar{x}\}$ with $x_{n} \rightarrow \bar{x}$ satisfying

$$
F_{\bar{x}}\left(x_{n}\right) \in-Q \backslash\{0\}(\forall n \geq 1) .
$$

We now consider the sequences $\left(v_{n}\right)_{\geq 1} \subset \operatorname{cone}(C-\bar{x})$ and $\left(t_{n}\right)_{n \geq 1} \subset(0,+\infty)$ with

$$
\begin{gathered}
v_{n}=\frac{x_{n}-\bar{x}}{\left\|x_{n}-\bar{x}\right\|}, n=1,2, \ldots, \\
t_{n}=\left\|x_{n}-\bar{x}\right\|, n=1,2, \ldots
\end{gathered}
$$

From the facts that $t_{n} \rightarrow 0^{+}$as $n \rightarrow+\infty,\left\|v_{n}\right\|=1$ for all $n \geq 1$, and moreover, $X$ is a finite dimensional space, we see that

$$
v_{n} \rightarrow v \in T(C ; \bar{x}) \backslash\{0\}
$$


Indeed, from the initial assumptions, $\operatorname{dim}(X)<+\infty$, which ensures that there exists a subsequence of $\left(\frac{x_{n}-\bar{x}}{\left\|x_{n}-\bar{x}\right\|}\right)_{n \geq 1}$ converging to $v$ with $\|v\|=1$. Also, the sequence $\left(\frac{x_{n}-\bar{x}}{\left\|x_{n}-\bar{x}\right\|}\right)_{n \geq 1}$ converges to $v$, which means that $v_{n} \rightarrow v$. On one hand, we get $x_{n}=\bar{x}+t_{n} v_{n} \in C$ for all $n \geq 1$ and $t_{n} \rightarrow 0^{+}$, which yield that $v \in T(C ; \bar{x}) \backslash\{0\}$. On the other hand, for each $n \in \mathbb{N}$, it follows from the hypotheses $\lambda \in Q^{+}$that

$$
\left\langle\lambda, F_{\bar{x}}\left(x_{n}\right)\right\rangle \leq 0
$$

In addition, from the relation $g\left(x_{n}\right) \in-S(n \geq 1)$ and the fact $\eta \in N\left(-S, g(\bar{x})\right.$ implies $\eta \in S^{+}$ and $\langle\eta, g(\bar{x})\rangle=0$, we obtain the following inequality

$$
\left\langle\eta, g\left(x_{n}\right)\right\rangle \leq 0 \text {. }
$$

Combining (3.12)-(3.13) with $x_{n}:=\bar{x}+t_{n} v_{n}(\forall n \geq 1)$, we obtain the following inequality

$$
\left\langle\lambda, F_{\bar{x}}\left(\bar{x}+t_{n} v_{n}\right)\right\rangle+\left\langle\eta, g\left(\bar{x}+t_{n} v_{n}\right)\right\rangle \leq 0 .
$$

Dividing both sides by $t_{n}^{m}>0$ and then taking the limit as $n \rightarrow+\infty$, we arrive at

$$
d_{S}^{m}\left(\lambda_{0} F_{\bar{x}}+\eta_{0} g\right)(\bar{x} ; v) \leq 0,
$$

which conflicts with (3.11). And we get the desired conclusion.

Remark 3.5. Considering the problem (VEPC), which is given as in Example 3.3, we deduce that $\bar{x}=(0,0,0)$ is a local efficient solution of problem (VEPC) because of $d_{S}^{m}\left(\lambda_{0} F_{\bar{x}}+\right.$ $\left.\eta_{0} g\right)(\bar{x} ; v)>0$ when $v \neq 0$. Furthermore, it should be noted here that Theorem 3.4 can not be employed with an efficient solution without the local property. An example is provided as follows.

Example 3.6. Let $X=Y=\mathbb{R}^{3}, Z=\mathbb{R}^{2}, C=[0,2 \pi] \times\left[0, \frac{5}{4} \pi\right] \times\left[0, \frac{3}{2} \pi\right], Q=\mathbb{R}_{+}^{3}, S=\mathbb{R}_{+}^{2}$, $\bar{x}=(0,0,0)$ and the functions $F_{\bar{x}}, g$ be defined respectively by

$$
\begin{aligned}
& F_{\bar{x}}\left(x_{1}, x_{2}, x_{3}\right)=\left(\sin x_{1}^{m}, \frac{1}{2} \sin x_{2}^{m}, \frac{1}{3} \sin x_{3}^{m}\right) \forall\left(x_{1}, x_{2}, x_{3}\right) \in C, \\
& g\left(x_{1}, x_{2}, x_{3}\right)=\left(-x_{1}^{m}-x_{2}^{m-1} x_{3}^{2},-x_{2}^{m}-x_{1}^{3} x_{3}^{m-2}\right) \forall\left(x_{1}, x_{2}, x_{3}\right) \in C .
\end{aligned}
$$

It can be easily seen that $Q^{+}=\mathbb{R}_{+}^{3}, N(-S, g(\bar{x}))=\mathbb{R}_{+}^{2}, T(C ; \bar{x})=\mathbb{R}_{+}^{3}$. In addition, for every $v=\left(v_{1}, v_{2}, v_{3}\right) \in \mathbb{R}_{+}^{3}, \lambda=\left(\lambda_{1}, \lambda_{2}, \lambda_{3}\right) \in Q^{+}$and $\eta=\left(\eta_{1}, \eta_{2}\right) \in N(-S, g(\bar{x}))$, the higher-order Studniarski derivatives $d_{S}^{m}\left(\lambda_{0} F_{\bar{x}}\right)(\bar{x} ; v)$ and $d_{S}^{m}\left(\eta_{0} g\right)(\bar{x} ; v)$ exist and are given as

$$
\begin{aligned}
& d_{S}\left(\lambda_{0} F_{\bar{x}}\right)(\bar{x} ; v)=\lambda_{1} v_{1}^{m}+\frac{1}{2} \lambda_{2} v_{2}^{m}+\frac{1}{3} \lambda_{3} v_{3}^{m}, \\
& d_{S}\left(\eta_{0} g\right)(\bar{x} ; v)=-\eta_{1} v_{1}^{m}-\eta_{2} v_{2}^{m} .
\end{aligned}
$$

For all $v=\left(v_{1}, v_{2}, v_{3}\right) \in T(C ; \bar{x}) \backslash\{0\}$, taking $\lambda=(2,4,3) \in Q^{+} \backslash\{(0,0,0)\}$ and $\eta=(1,1) \in$ $N(-S, g(\bar{x}))$, one obtains the following strict inequality

$$
d_{S}\left(\lambda_{0} F_{\bar{x}}+\eta_{0} g\right)(\bar{x} ; v)=v_{1}^{m}+v_{2}^{m}+v_{3}^{m}>0 .
$$

Taking account of Theorem 3.4, we conclude that $\bar{x}=(0,0,0)$ is a local efficient solution of problem (VEPC). In fact, in this setting, the feasible set of (VEPC) is of the form $K=C$, and moreover, $\sin x_{i}^{m} \geq 0$ for all $0 \leq x_{i} \leq \sqrt[m]{\pi}, i=1,2,3$. By taking $\delta=\sqrt[m]{\pi}$, we conclude that $F_{\bar{x}}\left(x_{1}, x_{2}, x_{3}\right) \notin-Q \backslash\{(0,0,0)\}$ for any $x=\left(x_{1}, x_{2}, x_{3}\right) \in K \cap B(\bar{x}, \delta)$, which means that $\bar{x}=(0,0,0)$ is a local efficient solution of problem (VEPC), as checked. 
It should be noticed in Example 3.6 that, the vector $\bar{x}=(0,0,0)$ is not an efficient solution to problem (VEPC). Indeed, we may choose a vector

$$
\tilde{x}=\left(\sqrt[m]{\frac{5}{4}} \pi, \sqrt[m]{\frac{5}{4}} \pi, \sqrt[m]{\frac{5}{4}} \pi\right) \in K
$$

By a direct computation, the following relation holds

$$
F_{\bar{x}}(\tilde{x})=\left(-\frac{\sqrt{2}}{2},-\frac{\sqrt{2}}{4},-\frac{\sqrt{2}}{6}\right) \in-Q \backslash\{(0,0,0)\},
$$

which entails that $\bar{x}$ can not belongs to an efficient solution of problem (VEPC).

\section{HighER-ORDER OPTIMALITY CONDITIONS FOR WEAK EFFICIENT SOLUTIONS, HENIG EFFICIENT SOLUTIONS, GLOBAL EFFICIENT SOLUTIONS AND SUPEREFFICIENT SOLUTIONS}

This section deals with the Kuhn-Tucker necessary and sufficient optimality conditions for the weak efficient, Henig efficient, global efficient and superefficient solutions of the vector equilibrium problems with constraints in terms of higher-order Studniarski's derivatives.

We begin with establishing Kuhn-Tucker necessary and sufficient optimality conditions for the weak efficient solution of problem (VEPC).

Theorem 4.1. (Higher-order necessary optimality condition) Let $\bar{x} \in K$ with $F_{\bar{x}}(\bar{x})=0$ be a weak efficient solution of problem (VEPC) and the interiors of $Q$ and $S$ be nonempty. Assume that there exists the Studniarski derivative of order $m$ of $\lambda_{0} F_{\bar{x}}\left(\forall \lambda \in Q^{+}\right)$and $\eta_{0} g(\forall \eta \in$ $N(-S, g(\bar{x})))$ at $\bar{x}$ in all the directions $v \in T(C ; \bar{x})$. Suppose also that the mapping $h_{\bar{x}}()=$. $\left(F_{\bar{x}}(),. g().\right): C \rightarrow Y \times Z$ is nearly $Q \times S$-subconvexlike on $C$ and the constraint qualification of the $(\mathrm{CQm})$ type is fulfilled. Then there exists $(\lambda, \eta) \in Y^{*} \times Z^{*}$ such that

$$
\begin{gathered}
\lambda \in Q^{+} \backslash\{0\}, \quad \eta \in N(-S, g(\bar{x})) ; \\
\min _{v \in T(C ; \bar{x})}\left\{d_{S}^{m}\left(\lambda_{0} F_{\bar{x}}+\eta_{0} g\right)(\bar{x} ; v)\right\}=0 .
\end{gathered}
$$

Proof. Let $\bar{x}$ be a weak efficient solution of problem (VEPC), that is,

$$
F_{\bar{x}}(K) \cap(-\operatorname{int} Q)=\emptyset .
$$

By invoking the property known in the book of convex analysis of Rockafellar [27], we have

$$
Q+\operatorname{int} Q=\operatorname{int} Q
$$

which together with (4.3) yields that

$$
\left(F_{\bar{x}}(K)+Q\right) \cap(-\operatorname{int} Q)=\emptyset .
$$

From (4.4) and the fact that $S+\operatorname{int} S \subset S$, one has

$$
\left(h_{\bar{x}}(C)+Q \times S\right) \cap(-\operatorname{int}(Q \times S))=\emptyset .
$$

In fact, if not, then there exists $x^{0} \in C$ such that from the following relation

$$
Q \times S+\operatorname{int}(Q \times S)=\operatorname{int}(Q \times S) \subset Q \times S,
$$


it leads to

$$
h_{\bar{x}}\left(x^{0}\right) \in-\operatorname{int}(Q \times S) .
$$

Hence, $g\left(x^{0}\right) \in-S$, which further yields that $x^{0} \in K$ and $F_{\bar{x}}\left(x^{0}\right) \in-i n t Q$. This is a contradiction. It should be mentioned here that the cones int $Q$ and int $S$ are open and convex. Combined this with (4.5), it ensures that

$$
\left(\operatorname{cl}\left(\operatorname{cone}\left(h_{\bar{x}}(C)+Q \times S\right)\right)\right) \cap(-\operatorname{int}(Q \times S))=\emptyset .
$$

From [9, Theorem 3.1] with the fact that $C_{U}(B)$ is replaced by $Q$, it follows that from Rockafellar's result [27] that there exists $(\lambda, \eta) \in Y^{*} \times Z^{*}$, not all zero, such that $\lambda \in Q^{+}$and $\eta \in N(-S, g(\bar{x}))$. Notice that $\lambda \neq 0$ is due to the proof of Theorem 3.1, which yields that (4.1) is valid. In addition, the inequality (3.5) also holds, that is,

$$
\left\langle\lambda, F_{\bar{x}}(x)\right\rangle+\langle\eta, g(x)\rangle \geq 0 \forall x \in C .
$$

Arguing similarly as for proving Theorem 3.1, we obtain the desired conclusion immediately.

The following example is proposed to illustrate the result obtained in Theorem 4.1.

Example 4.2. We consider the problem (VEPC), which is given as in Example 3.3 in which $Q=\mathbb{R}_{+}^{3}$ and $f_{3}(x)=x_{2}^{m}-x_{3}^{m}$ for all $x=\left(x_{1}, x_{2}, x_{3}\right) \in \mathbb{R}^{3}$. It is well-known that int $Q \neq \emptyset$, int $S \neq \emptyset$, $Q^{+}=\mathbb{R}_{+}^{3}$, and $\bar{x}=(0,0,0)$ is a weak efficient solution of problem (VEPC): Finding the vector $\bar{x} \in K$ such that

$$
F_{\bar{x}}(K) \cap(-\operatorname{int} Q)=\emptyset .
$$

Proceeding on the same lines as in Example 3.3, it yields an assertion that all of the hypotheses of Theorem 4.1 are fulfilled. In fact, in this setting, by taking

$$
\begin{aligned}
& \lambda=(2,1,2) \in \mathbb{R}_{+}^{3} \backslash\{(0,0,0)\}, \\
& \eta=(1,0) \in N(-S, g(\bar{x}))=\mathbb{R}_{+}^{2},
\end{aligned}
$$

the following result holds true

$$
\min _{v \in T(C ; \bar{x})}\left\{d_{S}^{m}\left(\lambda_{0} F_{\bar{x}}+\eta_{0} g\right)(\bar{x} ; v)\right\}=\min _{v \in \mathbb{R}_{+}^{3}}\left(v_{1}^{m}+v_{2}^{m-2} v_{3}^{2}\right)=0
$$

Remark 4.3. The result obtained in Theorem 4.1 is still true in the case that the nearly conesubconvexlike is removed and replaced by the cone-convexity (see 2.7 for more details). As a special case, we may assume $Y=\mathbb{R}^{p}, Z=\mathbb{R}^{q}, Q=\mathbb{R}_{+}^{p}$ and $S=\mathbb{R}_{+}^{q}$. Then these optimality condition is not changed in the case that the nearly cone-subconvexlike is replaced by the generalized convexity. In addition, the results in this remark are the same for the case that $\bar{x}$ is a local weak efficient solution to problem (VEPC).

Next, a sufficient optimality condition for a local weak efficient solution of problem (VEPC) will be derived.

Theorem 4.4. (Higher-order sufficient optimality condition) Let $\bar{x} \in K$ and assume that there exist the Studniarski derivative of order $m$ of $\lambda_{0} F_{\bar{x}}\left(\forall \lambda \in Q^{+}\right)$and $\eta_{0} g(\forall \eta \in N(-S, g(\bar{x})))$ at $\bar{x}$ in all the directions $v \in T(C ; \bar{x})$. Moreover, $\operatorname{dim} X<+\infty$. Suppose also that, for all $\forall v \in T(C ; \bar{x}) \backslash\{0\}$, there exist $\lambda \in Q^{+}$and $\eta \in N(-S, g(\bar{x}))$ satisfying

$$
d_{S}^{m}\left(\lambda_{0} F_{\bar{x}}+\eta_{0} g\right)(\bar{x} ; v)>0 .
$$


Then $\bar{x}$ is a local weak efficient solution of problem (VEPC).

Proof. Applying the result obtained in Theorem 3.4, we have that $\bar{x}$ is a local efficient solution of problem (VEPC). That is, there exists $\delta>0$ satisfying the following relation

$$
F_{\bar{x}}(K \cap B(\bar{x}, \delta)) \cap(-Q \backslash\{0\})=\emptyset .
$$

Therefore,

$$
F_{\bar{x}}(K \cap B(\bar{x}, \delta)) \cap(-\operatorname{int} Q)=\emptyset,
$$

which proves the claim.

In what follows, Kuhn-Tucker necessary and sufficient efficiency conditions for the rest efficient solutions of problem (VEPC) will be formulated.

Theorem 4.5. (Higher-order necessary optimality condition) Let the vector $\bar{x} \in K$ with $F_{\bar{x}}(\bar{x})=$ 0 and assume that there exist the Studniarski derivative of order $m$ of $\lambda_{0} F_{\bar{x}}\left(\forall \lambda \in Q^{+}\right)$and $\eta_{0} g(\forall \eta \in N(-S, g(\bar{x})))$ at $\bar{x}$ in all the directions $v \in T(C ; \bar{x})$. Suppose also that the mapping $h_{\bar{x}}()=.\left(F_{\bar{x}}(),. g().\right): C \rightarrow Y \times Z$ is nearly $Q \times S-$ subconvexlike on $C, Q$ has a base $B$, int $S \neq \emptyset$ and the constraint qualification of the (CQm) type is fulfilled. If $\bar{x}$ is a Henig efficient (resp., global efficient, superefficient if, in addition, $B$ is closed and bounded) solution of problem (VEPC), then there exist $(\lambda, \eta) \in Y^{*} \times Z^{*}$, not all zero, satisfying

$$
\begin{gathered}
\lambda \in Q^{\Delta}(B)\left(\text { resp. } Q^{\sharp}, \operatorname{int}\left(Q^{+}\right)\right), \eta \in N(-S, g(\bar{x})) ; \\
\min _{v \in T(C ; \bar{x})}\left\{d_{S}^{m}\left(\lambda_{0} F_{\bar{x}}+\eta_{0} g\right)(\bar{x} ; v)\right\}=0 .
\end{gathered}
$$

Proof. Let us first assume that $\bar{x}$ is a Henig efficient solution of problem (VEPC). Then there would exists some absolutely open convex neighborhood $U$ of the origin with $U \subset V_{B}$ such that

$$
F_{\bar{x}}(K) \cap(- \text { int cone }(U+B))=\emptyset .
$$

Taking into account the proof of Theorem 4.1, we deduce that

$$
\left(h_{\bar{x}}(C)+Q \times S\right) \cap(- \text { int cone }(U+B)) \times(- \text { int } S)=\emptyset .
$$

In addition, arguing similarly as for proving Theorem 4.1 with applying cone $(U+B)$ instead of $Q$, we have that there exists $(\lambda, \eta) \in Y^{*} \times Z^{*} \backslash\{(0,0)\}$ satisfying

$$
\begin{gathered}
\lambda \in[\operatorname{cone}(U+B)]^{+} \backslash\{0\}, \eta \in N(-S, g(\bar{x})), \\
\min _{v \in T(C ; \bar{x})}\left\{d_{S}^{m}\left(\lambda_{0} F_{\bar{x}}+\eta_{0} g\right)(\bar{x} ; v)\right\}=0 .
\end{gathered}
$$

It is well-known that the following inclusion holds (see $[4,5,9])$

$$
[\operatorname{cone}(U+B)]^{+} \backslash\{0\} \subset Q^{\Delta}(B) .
$$

So, $\lambda \in Q^{\Delta}(B)$, which means that conditions (4.8)-(4.9) are satisfied.

We next suppose that the vector $\bar{x} \in K$ is a global efficient solution of problem (VEPC). Then there exists a pointed convex cone $H \subset Y$ with $Q \backslash\{0\} \subset \operatorname{int} H$ satisfying

$$
F_{\bar{x}}(K) \cap(-H \backslash\{0\})=\emptyset,
$$

which entails that

$$
F_{\bar{x}}(K) \cap(-\operatorname{int} H)=\emptyset \text {. }
$$


Then, in a similar way to the proof of the first case with replacing the cone $Q$ by the cone $H$, we have that $(\lambda, \eta) \in Y^{*} \times Z^{*}$ with $\lambda \in H^{+} \backslash\{0\}, \eta \in N(-S, g(\bar{x}))$ satisfying (4.9). In addition, it is not difficult to see that $\lambda \in Q^{\sharp}$.

Finally, it should be mentioned here that the vector $\bar{x}$ is a superefficient solution of problem (VEPC) if and only if the vector $\bar{x}$ is a Henig efficient solution of problem (VEPC). Moreover, $\operatorname{int}\left(Q^{+}\right)=Q^{\Delta}(B)$. This helps us to end the proof.

In the same way as in the statement of Theorem 4.4, we have the following result.

Theorem 4.6. (Higher-order sufficient optimality conditions) Let $\bar{x} \in K$ and assume that there exist the Studniarski derivative of order $m$ of $\lambda_{0} F_{\bar{x}}\left(\forall \lambda \in Q^{+}\right)$and $\eta_{0} g(\forall \eta \in N(-S, g(\bar{x})))$ at $\bar{x}$ in all the directions $v \in T(C ; \bar{x}) ; Q$ has a base $B$ and $\operatorname{dim} X<+\infty$. Suppose also that, for all $\forall v \in T(C ; \bar{x}) \backslash\{0\}$, there exists $\lambda \in Q^{\Delta}(B)$ (resp. $Q^{\sharp}$, int $\left(Q^{+}\right)$) and $\eta \in N(-S, g(\bar{x})$ ) satisfying

$$
d_{S}^{m}\left(\lambda_{0} F_{\bar{x}}+\eta_{0} g\right)(\bar{x} ; v)>0 .
$$

Then $\bar{x}$ is a local Henig efficient (resp. local global efficient, local superefficient if, in addition, $B$ is closed and bounded) solution of problem (VEPC).

Proof. Let us first suppose that there exist $(\lambda, \eta) \in Y^{*} \times Z^{*}$ such that (4.8) and (4.10) hold. It suffices to show that the vector $\bar{x} \in C$ is a local Henig efficient solution to the problem (VEPC) in the sense $\lambda \in Q^{\Delta}(B)$. In fact, taking account of Gong's result $[4,5,9]$ together with $\lambda \in Q^{\Delta}(B)$, it ensures that there exists an open absolutely convex neighborhood $U$ of the origin in $Y$ with $U \subset V_{B}$ satisfying $\lambda \in[\text { cone }(U+B)]^{+} \backslash\{0\}$. Proceeding on the same lines as in the proof of Theorem 4.4, we conclude a conclusion that there exists a positive real number $\delta>0$ satisfying

$$
F_{\bar{x}}(K \cap B(\bar{x}, \delta)) \cap(- \text { int cone }(U+B))=\emptyset,
$$

which yields that

$$
\operatorname{cone}\left(F_{\bar{x}}(K \cap B(\bar{x}, \delta))\right) \cap(-\operatorname{int} \text { cone }(U+B))=\emptyset .
$$

We next consider the case $\lambda \in Q^{\sharp}$. By setting

$$
H=\{y \in Y:\langle\lambda, y\rangle>0\} \cup\{0\},
$$

we can conclude that $H$ is a pointed and convex cone satisfying

$$
Q \backslash\{0\} \subset \operatorname{int} H .
$$

In addition, it is evident that $\lambda \in H^{+}$, which provides (4.10). In view of the result obtained in Theorem 3.4, it leads to

$$
F_{\bar{x}}(K \cap B(\bar{x}, \delta)) \cap(-H \backslash\{0\})=\emptyset .
$$

This help us to understand that the vector $\bar{x}$ is a local global efficient solution to problem (VEPC), as required.

We finally consider the case $\lambda \in \operatorname{int}\left(Q^{+}\right)$. Note that the cone $Q$ has a bounded and closed base $B, \operatorname{int}\left(Q^{+}\right)=Q^{\Delta}(B)$ and the vector $\bar{x}$ is a local Henig efficient solution. Using the result obtained in the proof of the first case, we obtain the desired conclusion immediately.

Now, we give an example to illustrate Theorems 4.5 and 4.6. 
Example 4.7. Let $X=Y=Z=\mathbb{R}^{2}, C=\left\{(0,0),\left(-\frac{1}{\sqrt[m]{2}}, 1\right),(1,1),(1,0)\right\} \cup\left\{\left(0, \frac{1}{n}\right): n=1,2, \ldots\right\}$, $Q=S=\mathbb{R}_{+}^{2}$ and $\bar{x}=(0,0)$. Let $F_{\bar{x}}: \mathbb{R}^{2} \rightarrow \mathbb{R}^{2}$ be map defined by $F_{\bar{x}}=\left(f_{1}, f_{2}\right)$, where $f_{1}(x)=$ $x_{1}^{m}$ and $f_{2}(x)=x_{2}^{m}, \forall x=\left(x_{1}, x_{2}\right) \in \mathbb{R}^{2}$. Let $g: \mathbb{R}^{2} \rightarrow \mathbb{R}^{2}$ be a map defined by $g=\left(g_{1}, g_{2}\right)$, where $g_{1}(x)=-x_{1}^{m}-x_{2}^{m}$ and $g_{2}(x)=-x_{2}^{m}, \forall x=\left(x_{1}, x_{2}\right) \in \mathbb{R}^{2}$. By directly calculating, $Q^{+}=S^{+}=$ $N(-S, g(\bar{x}))=\mathbb{R}_{+}^{2}$ and $T(C ; \bar{x})=\{(0,0)\} \cup\left\{\left(0, \frac{1}{n}\right): n=1,2, \ldots\right\}$. Moreover, $Q$ has a closed and bounded base $B$, where

$$
B=\left\{\left(x_{1}, x_{2}\right) \in \mathbb{R}_{+}^{2}: x_{1}+x_{2}=1\right\} .
$$

Taking an absolutely convex neighborhood $U$ of the origin with $U \subset V_{B}$ with

$$
U=\left\{\left(x_{1}, u_{2}\right) \in \mathbb{R}^{2}: x_{1}^{2}+x_{2}^{2}<\frac{1}{2}\right\},
$$

we can check that the vector $\bar{x}=(0,0)$ is a Henig efficient solution, a globally efficient solution and a superefficient solution to problem (VEPC), the mapping $h_{\bar{x}}()=.\left(F_{\bar{x}}(),. g().\right): C \rightarrow Y \times Z$ is nearly $Q \times S$-subconvexlike on $C$, and the constraint qualification of the (CQm) type is fulfilled. Besides, it can be seen that there exists the Studniarski derivative of order $m$ of $\lambda_{0} F_{\bar{x}}\left(\forall \lambda \in Q^{+}\right)$ and $\eta_{0} g(\forall \eta \in N(-S, g(\bar{x})))$ at $\bar{x}$ in all the directions $v \in T(C ; \bar{x})$. So, all the hypotheses of Theorem 4.5 are valid. In this setting, by choosing $\lambda=(1,1) \in Q^{\Delta}(B)=\operatorname{int}\left(Q^{+}\right)=\mathbb{R}_{++}^{2}=$ $\operatorname{int}\left(\mathbb{R}_{+}^{2}\right)$ and $\eta=(0,0) \in N(-S, g(\bar{x}))$, it follows that

$$
\begin{gathered}
\min _{v \in T(C ; \bar{x})}\left\{d_{S}^{m}\left(\lambda_{0} F_{\bar{x}}+\eta_{0} g\right)(\bar{x} ; v)\right\}=\min _{v \in T(C ; \bar{x})}\left(v_{1}^{m}+v_{2}^{m}\right)=0, \\
\left.d_{S}^{m}\left(\lambda_{0} F_{\bar{x}}+\eta_{0} g\right)(\bar{x} ; v)\right\}=v_{1}^{m}+v_{2}^{m}>0 \text { for some } v \neq 0 .
\end{gathered}
$$

\section{THE APPLICATIONS TO A CONSTRAINED VECTOR OPTIMIZATION PROBLEM}

In this section, with the help of the results obtained in Sections 3 and 4, we can derive the Kuhn-Tucker necessary and sufficient optimality conditions via Studniarski's derivatives of higher order for the efficient solutions of a constrained vector optimization problem. A special case of (VEPC) is the constrained vector optimality problem (VOPC) involving

$$
F(x, y)=f(y)-f(x), \forall x, y \in X,
$$

where $f: X \rightarrow Y$ is a vector-valued mapping. A vector $\bar{x} \in K$ is a (local) efficient solution or a (local) weak efficient solution or a (local) Henig efficient solution or a (local) global efficient solution or a (local) superefficient solution of problem (VEPC) will be called a (local) efficient solution or a (local) weak efficient solution or a (local) Henig efficient solution or a (local) global efficient solution or a (local) superefficient solution of problem (VOPC), respectively (see $[4,5]$ or [9] for more details).

In the sequel, the result of necessary and sufficient optimality conditions for the efficient solutions of problem (VOPC) in terms of higher-order Studniarski's derivatives will be obtained.

Theorem 5.1. (Higher-order necessary optimality condition) Let $F_{x}(y)=f(y)-f(x)(\forall x, y \in$ $C)$ and let $\bar{x}$ be an efficient solution to the problem (VOPC). Assume that there exists the Studniarski derivative of order $m$ of $\lambda_{0} f\left(\forall \lambda \in Q^{+}\right)$and $\eta_{0} g(\forall \eta \in N(-S, g(\bar{x})))$ at $\bar{x}$ in all the directions $v \in T(C ; \bar{x})$. Suppose, furthermore, that qri $(f, g)(C) \neq \emptyset$, qri $(Q \times S) \neq \emptyset$ 
and cl cone $(q r i(c o(f, g)(C))+q r i(Q \times S))$ is not a linear subspace of $Y \times Z$. Then there exist $(\lambda, \eta) \in Y^{*} \times Z^{*} \backslash\{(0,0)\}$ satisfying

$$
\begin{gathered}
\lambda \in Q^{+}, \eta \in N(-S, g(\bar{x})) ; \\
\min _{v \in T(C ; \bar{x})}\left\{d_{S}^{m}\left(\lambda_{0} f+\eta_{0} g\right)(\bar{x} ; v)\right\}=0 .
\end{gathered}
$$

Moreover, $\lambda \neq 0$ under the constraint qualification of the (CQm) type.

Proof. It can be seen that, for all $t>0, \lambda \in Q^{+}$and $v \in X$, we have a representation as follows

$$
\frac{\lambda_{0} F_{\bar{x}}(\bar{x}+t v)-\lambda_{0} F(\bar{x})(\bar{x})}{t^{m}}=\frac{\lambda_{0} f(\bar{x}+t v)-\lambda_{0} f(\bar{x})}{t^{m}} .
$$

Thus, $d_{S}^{m}\left(\lambda_{0} f\right)(\bar{x} ; v)$ exists if and only if $d_{S}^{m}\left(\lambda_{0} F_{\bar{x}}\right)(\bar{x} ; v)$, too. We obtain the following equalities

$$
\begin{aligned}
d_{S}^{m}\left(\lambda_{0} f\right)(\bar{x} ; v) & =d_{S}^{m}\left(\lambda_{0} F_{\bar{x}}\right)(\bar{x} ; v) \\
\min _{v \in T(C ; \bar{x})}\left\{d_{S}^{m}\left(\lambda_{0} f+\eta_{0} g\right)(\bar{x} ; v)\right\} & =\min _{v \in T(C ; \bar{x})}\left\{d_{S}^{m}\left(\lambda_{0} F_{\bar{x}}+\eta_{0} g\right)(\bar{x} ; v)\right\} .
\end{aligned}
$$

In other words, we have the assertions that qri $(f, g)(C) \neq \emptyset$ if and only if qri $\left(F_{\bar{x}}, g\right)(C) \neq \emptyset$ and $c l$ cone $(\operatorname{qri}(c o(f, g)(C))+\operatorname{qri}(Q \times S))$ is not a linear subspace of the product space $Y \times Z$ that implies $c l$ cone $\left(\operatorname{qri}\left(\operatorname{co}\left(F_{\bar{x}}, g\right)(C)\right)+\operatorname{qri}(Q \times S)\right)$ is not a linear subspace either. Making use of the result of Theorem 3.1, we get the desired conclusion.

Theorem 5.2. (Higher-order sufficient optimality condition) Let $F_{x}(y)=f(y)-f(x)(\forall x, y \in C)$ and let $\bar{x}$ be a feasible point to the problem (VOPC). Assume that there exist the Studniarski derivatives of order $m$ of $\lambda_{0} f\left(\forall \lambda \in Q^{+}\right)$and $\eta_{0} g(\forall \eta \in N(-S, g(\bar{x})))$ at $\bar{x}$ in all the directions $v \in T(C ; \bar{x})$. Moreover, $\operatorname{dim} X<+\infty$. Furthermore, suppose that, for all $v \in T(C ; \bar{x}) \backslash\{0\}$, there exist $\lambda \in Q^{+}$and $\eta \in N(-S, g(\bar{x}))$ satisfying

$$
d_{S}^{m}\left(\lambda_{0} f+\eta_{0} g\right)(\bar{x} ; v)>0
$$

Then $\bar{x}$ is a local efficient solution of problem (VOPC).

Proof. Observe that the Studniarski derivative $d_{S}^{m}\left(\lambda_{0} F_{\bar{x}}\right)(\bar{x} ; v)$ exists and they are given as $d_{S}^{m}\left(\lambda_{0} f+\right.$ $\left.\eta_{0} g\right)(\bar{x} ; v)=d_{S}^{m}\left(\lambda_{0} F_{\bar{x}}+\eta_{0} g\right)(\bar{x} ; v)$ for all $v \in T(C ; \bar{x})$. Following the proof in Theorem 3.4, we obtain the desired conclusion easily.

By use of the nearly cone-subconvexlikeness, we have the following result.

Theorem 5.3. (Higher-order necessary optimality condition) Let $F_{x}(y)=f(y)-f(x) \quad(\forall x, y \in$ $C)$ and let $\bar{x} \in K$ be a weak efficient solution of problem (VOPC). Assume that there exist the Studniarski derivative of order $m$ of $d_{S}^{m}\left(\lambda_{0} f\right)(\bar{x} ; v)\left(\forall \lambda \in Q^{+}\right)$and $d_{S}^{m}\left(\eta_{0} g\right)(\bar{x} ; v)(\forall \eta \in$ $N(-S, g(\bar{x})))$ at $\bar{x}$ in all the directions $v \in T(C ; \bar{x})$. Suppose, furthermore, that the mapping $h_{\bar{x}}()=.(f()-.f(\bar{x}), g()):. C \rightarrow Y \times Z$ is nearly $Q \times S-$ subconvexlike on $C$, int $Q \neq \emptyset$, int $S \neq \emptyset$ and the constraint qualification of the (CQ1) type is valid. Then there exists $(\lambda, \eta) \in Y^{*} \times Z^{*}$, not all zero, satisfying

$$
\begin{gathered}
\lambda \in Q^{+} \backslash\{0\}, \eta \in N(-S, g(\bar{x})) ; \\
\min _{v \in T(C ; \bar{x})}\left\{d_{S}^{m}\left(\lambda_{0} f+\eta_{0} g\right)(\bar{x} ; v)\right\}=0 .
\end{gathered}
$$

Proof. From Theorem 5.1, we can obtain the desired conclusion immediately. 
Next, we obtain a sufficient optimality condition for the local weak efficient solution of problem (VOPC).

Theorem 5.4. (Higher-order sufficient optimality condition) Let $F_{x}(y)=f(y)-f(x) \quad(\forall x, y \in$ $C)$ and let $\bar{x}$ be a feasible point of problem (VOPC). Assume that there exist the Studniarski derivative of order $m$ of $\lambda_{0} f\left(\forall \lambda \in Q^{+}\right)$and $\eta_{0} g(\forall \eta \in N(-S, g(\bar{x})))$ at $\bar{x}$ in all the directions $v \in T(C ; \bar{x})$. Moreover, $\operatorname{dim} X<+\infty$. Suppose, furthermore, that for all $v \in T(C ; \bar{x}) \backslash\{0\}$ there exist $\lambda \in Q^{+}$and $\eta \in N(-S, g(\bar{x}))$ satisfying

$$
d_{S}^{m}\left(\lambda_{0} f+\eta_{0} g\right)(\bar{x} ; v)>0 .
$$

Then $\bar{x}$ is a local weak efficient solution of problem (VOPC).

Proof. From Theorem 5.2, we can obtain the desired conclusion immediately.

In the case that an ordering cone in the objective space has a base, we can derive a necessary optimality condition for the remain efficient solutions of problem (VOPC).

Theorem 5.5. (Higher-order necessary optimality conditions) Let the convex set $B$ be a base of a cone $Q, F_{x}(y)=f(y)-f(x)(\forall x, y \in C)$ and let $\bar{x} \in K$ be a Henig efficient (resp., global efficient, superefficient if, in addition, $B$ is closed and bounded) solution of problem (VOPC). Assume that there exist the Studniarski derivative of order $m$ of $\lambda_{0} F_{\bar{x}}\left(\forall \lambda \in Q^{+}\right)$and $\eta_{0} g(\forall \eta \in$ $N(-S, g(\bar{x})))$ at $\bar{x}$ in all the directions $v \in T(C ; \bar{x})$. Suppose also that the mapping $h_{\bar{x}}()=$. $(f()-.f(\bar{x}), g()):. C \rightarrow Y \times Z$ is nearly $Q \times S-$ subconvexlike on $C$, int $S \neq \emptyset$ and $(\mathrm{CQm})$ holds. Then there exists $(\lambda, \eta) \in Y^{*} \times Z^{*}$, not all zero, satisfying

$$
\begin{gathered}
\lambda \in Q^{\Delta}(B)\left(\text { resp. } Q^{\sharp}, \operatorname{int}\left(Q^{+}\right)\right), \eta \in N(-S, g(\bar{x})) ; \\
\min _{v \in T(C ; \bar{x})}\left\{d_{S}^{m}\left(\lambda_{0} f+\eta_{0} g\right)(\bar{x} ; v)\right\}=0 .
\end{gathered}
$$

Proof. From Theorem 4.5, we can obtain the desired conclusion immediately.

Finally, we give a sufficient optimality condition for the problem (VOPC).

Theorem 5.6. (Higher-order sufficient optimality conditions) Let the convex set $B$ be a base of $Q, F_{x}(y)=f(y)-f(x)(\forall x, y \in C)$ and $\bar{x}$ be a feasible point of problem (VOPC). Assume that there exist the Studniarski derivative of order $m$ of $\lambda_{0} F_{\bar{x}}\left(\forall \lambda \in Q^{+}\right)$and $\eta_{0} g(\forall \eta \in$ $N(-S, g(\bar{x})))$ at $\bar{x}$ in all the directions $v \in X$. Moreover, dimX $<+\infty$. Suppose also that, for all $v \in T(C ; \bar{x}) \backslash\{0\}$, there exist $\lambda \in Q^{\Delta}(B)$ (resp. $Q^{\sharp}$, int $\left(Q^{+}\right)$) and $\eta \in N(-S, g(\bar{x})$ ) satisfying

$$
d_{S}^{m}\left(\lambda_{0} f+\eta_{0} g\right)(\bar{x} ; v)>0 \text {. }
$$

Then $\bar{x}$ is a local Henig efficient (resp. local global efficient, local superefficient if, in addition, $B$ is closed and bounded) solution of problem (VOPC).

Proof. From Theorem 4.6, we can obtain the desired conclusion immediately.

Remark 5.7. The results obtained above are not changed in the sense that the nearly conesubconvexlike is removed and replaced by the cone-convexity.

To close this section, we take an example about the model of Nash-Cournot equilibria problem, which can be found in [30]. 
Example 5.8. (Nash-Cournot equilibria problem) Assume that there are $n$ companies, and each company $i$ possess $I_{i}$ generating units. Let $x$ denote the vector whose entry $x_{j}$ stands for the power generating by unit $j$. Suppose that the price $p_{i}(s)$ is a decreasing affine function of $s$ with $s=\sum_{i=1}^{N} x_{i}$, and $N$ the number of all generating units, i.e., $p_{i}(s)=\alpha-\beta_{i} s$. Then the profit made by company $i$ is

$$
f_{i}(x)=-\sum_{j \in I_{i}} c_{j}\left(x_{j}\right)+p_{i}(s) \sum_{j \in I_{i}} x_{j}
$$

where $c_{j}\left(x_{j}\right)$ is the cost for generating $x_{j}$ by generating unit $j$. Let us suppose that $K_{i}$ is the strategy set of company $i$, which means that $\sum_{j \in I_{i}} x_{j} \in K_{i}$ must be satisfied for all $i$. Then the strategy set of the model is of the form $K=K_{1} \times K_{2} \ldots \times K_{n}$. We recall that $\bar{x} \in K=K_{1} \times$ $K_{2} \ldots \times K_{n}$ is said to be an equilibrium point of the model if $f_{i}(\bar{x}) \geq f_{i}\left(\bar{x}\left[x_{i}\right]\right) \forall x_{i} \in K_{i}, \forall i=$ $1,2, \ldots, n$, where $\bar{x}\left[x_{i}\right]$ signifies the vector obtained from $\bar{x}$ by replacing $\bar{x}_{i}$ with $x_{i}$. We take $F(x, y):=\Psi(x, y)-\Psi(x, x)$, with $\Psi(x, y):=-\sum_{i=1}^{n} f_{i}\left(x\left[y_{i}\right]\right)$. Without of loss generality, we can suppose that the price $p_{i}($.$) is a nonnegative function and then setting g_{i}():.=-p_{i}($.$) and$ $K_{0}:=K \cap\left\{x \in K: g_{i}(x) \leq 0 \forall i=1, \ldots, N\right\}$. Then the problem of finding a Nash equilibrium point of the model can be formulated: Finding $\bar{x} \in K_{0}$ such that

$$
F_{\bar{x}}(x) \geq 0 \forall x \in K_{0} .
$$

Assume that there exists the Studniarski derivative of order 1 of $c_{i}$ at $\bar{x}_{i}(i=1, \ldots, N)$ in all the directions $v \in \mathbb{R}^{N}$. Then there exist the Studniarski derivative of order 1 of $F_{\bar{x}}()=.F(\bar{x},$.$) and$ $g=\left(g_{1}, g_{2}, \ldots, g_{N}\right)$ at $\bar{x}$ in all the directions $v \in \mathbb{R}^{N}$, and given respectively by

$$
\begin{aligned}
d_{S} F_{\bar{x}}(\bar{x} ; x-\bar{x}) & =\sum_{i=1}^{n} \sum_{j \in I_{i}} d_{S} c_{j}\left(\bar{x}_{j} ; x_{j}-\bar{x}_{j}\right)+\sum_{i=1}^{n} \sum_{j \in I_{i}} \beta_{i}\left(\sum_{k=1}^{N} x_{k} \bar{x}_{k}+\bar{x}_{j}\left(x_{j}-\bar{x}_{j}\right)\right) \\
& -\sum_{i=1}^{n} \sum_{j \in I_{i}} \beta_{i} \bar{x}_{j} \sum_{k=1}^{N}\left(x_{k}-\bar{x}_{k}\right)-\sum_{i=1}^{n} \sum_{j \in I_{i}} \alpha\left(x_{j}-\bar{x}_{j}\right), \\
d_{S} g(\bar{x} ; x-\bar{x}) & =\left(\beta_{1} \sum_{i=1}^{N}\left(x_{i}-\bar{x}_{i}\right), \beta_{2} \sum_{i=1}^{N}\left(x_{i}-\bar{x}_{i}\right), \ldots, \beta_{N} \sum_{i=1}^{N}\left(x_{i}-\bar{x}_{i}\right)\right)
\end{aligned}
$$

for all $x=\left(x_{1}, x_{2}, \ldots, x_{N}\right) \in \mathbb{R}_{+}^{N}$. We pick $\bar{x}=\left(\bar{x}_{1}, \bar{x}_{2}, \ldots, \bar{x}_{N}\right) \in K$. Suppose that the strict inequality $\sum_{i=1}^{N} \bar{x}_{i}>0$ is valid. In addition, the constraint qualification of the (CQ1) type holds. In this sense, if $\bar{x} \in K_{0}$ is a weak efficient solution of (EP), then there exist $\bar{\lambda}>0$ and $\bar{\eta} \geq 0$ with $\bar{\eta}(g(\bar{x}))=0$ satisfying

$$
\begin{aligned}
\bar{\lambda} & {\left[\sum_{i=1}^{n} \sum_{j \in I_{i}} d_{S} c_{j}\left(\bar{x}_{j} ; x_{j}\right)+\sum_{i=1}^{n} \sum_{j \in I_{i}} \beta_{i}\left(\sum_{k=1}^{N} x_{k} \bar{x}_{k}+\bar{x}_{j} x_{j}\right)\right.} \\
& \left.-\sum_{i=1}^{n} \sum_{j \in I_{i}} \beta_{i} \bar{x}_{j} \sum_{k=1}^{N} x_{k}-\sum_{i=1}^{n} \sum_{j \in I_{i}} \alpha x_{j}\right]+\sum_{j=1}^{N} \sum_{i=1}^{N} \bar{\eta}_{j} \beta_{j} x_{i} \geq 0
\end{aligned}
$$


for all $x=\left(x_{1}, x_{2}, \ldots, x_{N}\right) \in T\left(\mathbb{R}_{+}^{N} ; \bar{x}\right)$, or equivalently,

$$
\begin{aligned}
\min _{x \in T\left(\mathbb{R}_{+}^{N} ; \bar{x}\right)}\{\bar{\lambda} & {\left[\sum_{i=1}^{n} \sum_{j \in I_{i}} d_{S} c_{j}\left(\bar{x}_{j} ; x_{j}\right)+\sum_{i=1}^{n} \sum_{j \in I_{i}} \beta_{i}\left(\sum_{k=1}^{N} x_{k} \bar{x}_{k}+\bar{x}_{j} x_{j}\right)\right.} \\
& \left.\left.-\sum_{i=1}^{n} \sum_{j \in I_{i}} \beta_{i} \bar{x}_{j} \sum_{k=1}^{N} x_{k}-\sum_{i=1}^{n} \sum_{j \in I_{i}} \alpha x_{j}\right]+\sum_{j=1}^{N} \sum_{i=1}^{N} \bar{\eta}_{j} \beta_{j} x_{i}\right\}=0 .
\end{aligned}
$$

We close this paper by making some comparisons among the results obtained in the paper and the existing one in the literature.

Remark 5.9. Up to our knowledge, there are no results on higher-order necessary and sufficient optimality conditions in terms of Studniarski's derivatives in infinite-dimensional spaces for the efficient solutions of the vector equilibrium problems with constraints (VEPC). In this paper, we dealt with the higher-order necessary and sufficient conditions for the efficient solution types of problem (VEPC), while Studniarski [18] obtained only the higher-order optimality condition for the minimizers of multiobjective optimization problem and Luu [15] provided only the higherorder necessary and sufficient for the strict local pareto minima of multiobjective optimization problem via Studniarski's derivatives.

\section{Conclusions}

Higher-order optimality condition for the efficient solutions of vector equilibrium problems with constraints have been intensively investigated recently. By making use of the notation of the Studniarski derivative of higher order for the objective and constraint functions, we established the Fritz John and Kuhn-Tucker necessary and sufficient optimality conditions for efficient, weak efficient, Henig efficient, global efficient and superefficient solutions to problems (VEPC) and (VOPC). The results obtained in this paper have not been yet fully considered and moreover, these optimality conditions may be used to construct algorithms for finding efficient solutions of the vector equilibrium problems with constraints in the future.

\section{Funding}

The second author was funded by Vietnam National Foundation for Science and Technology Development (NAFOSTED) under Grant No. 101.01-2017.301.

\section{Acknowledgements}

The authors thank to the referees for their valuable comments and suggestions, which improved the paper in its present form.

\section{REFERENCES}

[1] E. Constantin, Higher order necessary conditions in smooth contrained optimization, Commun. Math. 479 (2009), 41-49.

[2] E. Constantin, Second-order optimality conditions for problems with locally Lipschitz data via tangential directions, Comm. Appl. Nonlinear Anal. 18 (2011), 75-84.

[3] Z. Hong, L. Jiao, D.S. Kim, On a class of nonsmooth fractional robust multi-objective optimization problems. Part I: Optimality conditions, Appl. Set-Valued Anal. Optim. 2 (2020), 109-121.

[4] X. H. Gong, Scalarization and optimality conditions for vector equilibrium problems, Nonlinear Anal. 73 (2010), 3598-3612. 
[5] X. H. Gong, Optimality conditions for vector equilibrium problems, J. Math. Anal. Appl. 342 (2008), 14551466.

[6] X. H. Gong, Optimality conditions for Henig and globally proper efficient solutions with ordering cone has empty interior, J. Math. Anal. Appl. 307 (2005), 12-31.

[7] X. H. Gong, Efficiency and Henig efficiency for vector equilibrium problems, J. Optim. Theory Appl. 108 (2001), 139-154.

[8] L.T. Tung, Karush-Kuhn-Tucker optimality conditions for nonsmooth multiobjective semidefinite and semiinfinite programming, J. Appl. Numer. Optim. 1 (2019), 63-75.

[9] X. J. Long, Y. Q. Huang, Z. Y. Peng, Optimality conditions for the Henig efficient solution of vector equilibrium problems with constraints, Optim. Letter. 5 (2011), 717-728.

[10] D. V. Luu, D. D. Hang, Efficient solutions and optimality conditions for vector equilibrium problems, Math. Meth. Oper. Res. 79 (2014), 163-177.

[11] D.V. Luu, D. D. Hang, On efficiency conditions for nonsmooth vector equilibrium problems with equilibrium constraints, Numer. Funct. Anal. Optim. 36 (2015), 1622-1642.

[12] Q. S. Qiu, Optimality conditions for vector equilibrium problems with constraints, J. Ind. Manag. Optim. 5 (2009), 783-790.

[13] D.V. Luu, Optimality conditions for local efficient solutions of vector equilibrium problems via convexificators and applications, J. Optim. Theory. Appl. 171 (2016), 643-665.

[14] D. V. Luu, Second-order necessary efficiency conditions for nonsmooth vector equilibrium problems, J. Glob. Optim. 70 (2018), 437-453.

[15] D. V. Luu, Higher-order necessary and sufficient conditions for strict local Pareto minima in terms of Studniarski's derivatives, Optim. 57 (2008), 593-605.

[16] H.T.H. Diem, P.Q. Khanh, L.T. Tung, On higher-order sensitivity analysis in nonsmooth vector optimization, J. Optim. Theory Appl. 162 (2014), 463-488.

[17] N. L. H. Anh, P. Q. Khanh, L.T. Tung, Higher-order radial derivatives and optimality conditions in nonsmooth vector optimization, Nonlinear Anal. 74 (2011), 7365-7379.

[18] M. Studniarski, Necessary and sufficient conditions for isolated local minima of nonsmooth functions, SIAM J. Control Optim. 24 (1986), 1044-1049.

[19] T.V. Su, N. D. Hien, Studniarski's derivatives and efficiency conditions for constrained vector equilibrium problems with applications, Optim. (2019), https://doi.org/10.1080/02331934.2019.1702985

[20] T.V. Su, D.D. Hang, Optimality conditions for the efficient solutions of vector equilibrium problems with constraints in terms of directional derivatives and applications, Bull. Iran. Math. Soc. 45 (2019), 1619-1650.

[21] D.V. Luu, T.V. Su, Contingent derivatives and necessary efficiency conditions for vector equilibrium problems with constraints, RAIRO - Oper. Res. 52 (2018), 543-559.

[22] Q.H. Ansari, I.V. Konnov, J.C. Yao, Existence of a Solution and Variational Principles for Vector Equilibrium Problems, J. Optim. Theory. Appl. 110(3) (2001), 481-492.

[23] Q.H. Ansari, X,Q. Yang, J.C. Yao, Existence and Duality of Implicit Vector Variational Problems, Numer. Funct. Anal. Optim. 22 (2001), 815-829.

[24] Q.H. Ansari, X.Q. Yang, J.C. Yao, Characterizations of Solutions for Vector Equilibrium Problems, J. Optim. Theory. Appl. 113 (2002), 435-447.

[25] J. M. Borwein, A. Lewis, Partially-finite convex programming, Par1: Quasirelative interiors and duality theory, Math. Program. 57 (1992), 15-48.

[26] F. Cammaroto, B. Di Bella, Separation theorem based on the quasirelative interior and application to duality theory, J. Optim. Theory Appl. 125 (2005), 223-229.

[27] R.T. Rockafellar, Convex Analysis. Princeton University Press, Princeton, 1970.

[28] B. Jiménez, V. Novo, First order optimality conditions in vector optimization involving stable functions, Optim. 57 (2008), 449-471.

[29] G. Giorgi, A. Guerraggio, On the notion of tangent cone in mathematical programming, Optim. 25 (1992), 11-23.

[30] L.H. Yen, L.D. Muu, N.T.T. Huyen, An algorithm for a class of split feasibility problems: application to a model inelectricity production, Math. Methods Oper. Res. 84 (2016), 549-565. 